A-16-2020

\title{
UTILIZACIÓN DEL CÓDIGO HYDRUS-2D PARA LA DETERMINACIÓN DE LA PROFUNDIDAD Y FRECUENCIA DE RIEGO EN ARROZ REGADO POR GOTEO SUBTERRÁNEO EN SUELOS DE DISTINTA TEXTURA
}

\author{
G. Arbat (1) (P), S. Cufí (2), M. Duran-Ros (3), J. Pinsach (4), C. Prieto (5), J. Pujol (6), J. \\ Puig-Bargués (7), F. Ramírez de Cartagena (8)
}

\begin{abstract}
1 Profesor Agregado, Universidad de Girona, Departamento de Ingeniería Química, Agraria y Tecnología Agroalimentaria, gerard.arbat@udg.edu

2 Becaria en formación, Universidad de Girona, Departamento de Ingeniería Química, Agraria y Tecnología Agroalimentaria, silvia97ca@gmail.com

${ }^{3}$ Profesor Agregado Interino, Universidad de Girona, Departamento de Ingeniería Química, Agraria y Tecnología Agroalimentaria, miquel.duranros@udg.edu

${ }^{4}$ Profesor Asociado, Universidad de Girona, Departamento de Ingeniería Química, Agraria y Tecnología Agroalimentaria, jaume.pinsach@udg.edu

5 Becaria en formación, Universidad de Girona, Departamento de Ingeniería Química, Agraria y Tecnología Agroalimentaria, cristina.prieto@udg.edu

6 Profesor Agregado, Universidad de Girona, Departamento de Ingeniería Química, Agraria y Tecnología Agroalimentaria, joan.pujol@udg.edu

${ }^{7}$ Profesor Titular de Universidad, Universidad de Girona, Departamento de Ingeniería Química, Agraria y Tecnología Agroalimentaria, jaume.puig@udg.edu

${ }^{8}$ Catedrático de Universidad, Universidad de Girona, Departamento de Ingeniería Química, Agraria y Tecnología Agroalimentaria, francisco.ramirez@udg.edu
\end{abstract}

\section{1- Introducción}

El arroz es uno de los principales cultivos a nivel mundial, siendo básico para la alimentación humana en muchos países. España es el segundo productor europeo después de Italia, con una superficie total de 105.422 ha en 2019 (MAPA, 2020). Tradicionalmente el arroz se cultiva en riego inundado durante prácticamente todo su ciclo y consume entre un 30 y un $40 \%$ del agua dulce a nivel mundial (Humphreys et al.,2010).

La escasez de agua representa una preocupación creciente, tanto en las zonas arroceras españolas como en otras zonas productoras del mundo (Zheng et al., 2020). Por ello, la utilización de técnicas de riego que permitan producir arroz con menor cantidad de agua cada vez tiene mayor interés. En este sentido, el riego por goteo subterráneo (RGS) es el sistema que permite un mayor ahorro de agua, mejorando las producciones cuando se compara con otras técnicas de cultivo de arroz aeróbico (Parthasarathi et al., 2018). Sin embargo, existen muy pocos trabajos publicados sobre el RGS en arroz y su aplicación es un reto importante (Rajwade et al., 2018). Prácticamente no existe información sobre la profundidad y separación más adecuada a la que deben instalarse los laterales, la separación y caudal de los emisores, así como la duración del riego. Es necesario por tanto establecer criterios para diseñar el sistema y manejar el riego de forma que se maximice la producción y minimicen las pérdidas de agua.

El modelo HYDRUS-2D ha sido aplicado con buenos resultados en alfalfa para optimizar la profundidad a que deben instalarse los laterales de riego en RGS, estableciendo diferentes escenarios de pauta de riego y textura del suelo (Reyes-Esteves y Slack, 2019). La versión unidimensional de este código, HYDRUS-1D ha demostrado poder predecir de forma 
adecuada el potencial de agua en el suelo, la evapotranspiración y la percolación profunda en arroz regado por inundación (Li et al., 2014).

\section{2- Objetivos}

El objetivo general del presente trabajo ha sido determinar la profundidad a la que deben instalarse los laterales y establecer la frecuencia de riego más adecuada en riego de arroz con RGS en suelos de distinta textura en la zona del Baix Ter (Girona), a partir de ensayos de campo y modelación con HYDRUS-2D.

\section{3- Materiales y Métodos}

\section{1.- Ensayo de campo}

Los ensayos de campo se llevaron a cabo en Pals (Girona, España) durante la temporada de cultivo de 2019 en una parcela experimental de 0,39 ha. La textura de la parcela, resultó ser espacialmente heterogénea, predominando la clase textural francoarenosa en el $85 \%$ de la superficie y la franca en el 15\% (Tabla 1). Además, se caracterizó otro suelo de textura arcillo-limosa (Tabla 1), representativo también de la zona de producción, con el objetivo de extrapolar mediante HYDRUS-2D los resultados obtenidos en el suelo del ensayo de campo.

Se instaló un sistema de RGS, con los laterales enterrados a $15 \mathrm{~cm}$ de profundidad y $66 \mathrm{~cm}$ de separación. Se utilizó tubería integral de goteo de pared delgada $(0,38 \mathrm{~mm}), 16 \mathrm{~mm}$ de diámetro interior y emisores autocompensantes de $1 \mathrm{~L} / \mathrm{h}$ (Dripnet PC ${ }^{\mathrm{TM}}$, Netafim, Israel), distanciados $0,3 \mathrm{~m}$.

La parcela se sembró en seco con la variedad Onice (28/5/2019) mediante sembradora de líneas a una dosis de $135 \mathrm{~kg} / \mathrm{ha}$ y separación de $19 \mathrm{~cm}$ entre líneas de siembra.

El criterio general de riego, se basó en mantener un potencial de $-10 \mathrm{kPa}$ a $10 \mathrm{~cm}$ de profundidad y a una distancia horizontal de $8 \mathrm{~cm}$ del emisor (Figura 1).

Tabla 1. Principales características físicas del suelo de la parcela experimental y de otra representativa de la zona, considerados en las simulaciones.

\begin{tabular}{|c|c|c|c|}
\hline \multirow{2}{*}{ Característica } & \multicolumn{2}{|c|}{ Parcela experimental } & \multirow{2}{*}{$\begin{array}{c}\begin{array}{c}\text { Otra parcela } \\
\text { característica } \\
\text { en la zona }\end{array} \\
\begin{array}{c}\text { Textura } \\
\text { Arcillo-limosa }\end{array}\end{array}$} \\
\hline & $\begin{array}{c}\text { Textura } \\
\text { Franco-arenosa }\end{array}$ & $\begin{array}{l}\text { Textura } \\
\text { Franca }\end{array}$ & \\
\hline Área de la parcela (\%) & 85,0 & 15,0 & - \\
\hline Arena (\%) & 64,9 & 47,4 & 6,5 \\
\hline Limo (\%) & 21,6 & 35,5 & 53,4 \\
\hline Arcilla (\%) & 13,5 & 17,1 & 40,1 \\
\hline Densidad aparente $\left(\mathrm{g} / \mathrm{cm}^{3}\right)$ & 1,53 & 1,45 & 1,26 \\
\hline Contenido de agua a $-33 \mathrm{kPa}$ & 16,8 & 26,1 & 44,1 \\
\hline Contenido de agua a $-1500 \mathrm{kPa}$ & 6,1 & 13,1 & 25,2 \\
\hline
\end{tabular}

La temperatura, pluviometría, presión atmosférica, radiación global y velocidad del viento se midieron con una estación meteorológica automatizada, situada aproximadamente a $2 \mathrm{~km}$ de la parcela experimental. La evapotranspiración de referencia $\left(E T_{0}\right)$ se determinó a partir del método de FAO 56 (Allen et al., 2006). El agua de riego aplicada en la parcela se midió en continuo mediante un contador volumétrico. El contenido de agua del suelo se determinó en continuo desde el día 34 después de la siembra (DDS), a 10 y $25 \mathrm{~cm}$ de profundidad y en dos posiciones relativas respecto al lateral de riego, una próxima al emisor a $8 \mathrm{~cm}$ del lateral y la otra en la zona intermedia entre 2 laterales de riego (Figura 1). Para ello se utilizó un sensor basado en la reflectometría en el dominio de la frecuencia, con una 
precisión de $\pm 3 \%$ del contenido volumétrico de agua en el suelo (SM 150-T, Delta-T Devices, Cambridge, Reino Unido). Los sensores estaban situados en la parte de la parcela con textura franco-arenosa. La profundidad del freático se monitorizó con lecturas semanales en 3 tubos piezométricos de $2 \mathrm{~m}$ de longitud situados alrededor de la parcela.

A lo largo de la campaña se realizó un seguimiento de los estadios fenológicos del cultivo. La producción se obtuvo a partir de un muestreo realizado el 18/10/2020, en el estadio de madurez fisiológica. Se tomaron 6 muestras en una superficie de referencia de $0,5 \times 0,5$ $\mathrm{m}^{2}$, correspondientes a las dos texturas presentes en la parcela. Después de secar las muestras se obtuvo la producción estandarizada a una humedad del grano del $14 \%$. Se determinó la producción media en las superficies correspondientes a cada clase textural, realizando un análisis de la varianza para averiguar la existencia de diferencias significativas en función del tipo de suelo.

La productividad del agua (WP), que caracteriza la producción por unidad de agua utilizada, se calculó de estas dos maneras:

$$
\begin{aligned}
& W P_{R+L L}=\frac{P}{R+L l} \\
& W P_{R}=\frac{P}{R}
\end{aligned}
$$

en la que $P$ representa la producción de arroz al $14 \%$ de humedad $(\mathrm{kg} / \mathrm{ha}) ; R$ el agua de riego aplicada $\left(\mathrm{m}^{3} / \mathrm{ha}\right)$ y $\mathrm{L} /$ la precipitación durante el periodo de cultivo $\left(\mathrm{m}^{3} / \mathrm{ha}\right)$.

\section{2.- Simulaciones de la dinámica de agua en el suelo}

Para modelar la dinámica del agua en el suelo se utilizó el código HYDRUS 2D (Šimůnek et al., 2008), que resuelve numéricamente la ecuación de Richards:

$$
\frac{\partial \theta}{\partial t}=\frac{\partial}{\partial x}\left[K(h) \frac{\partial h}{\partial x}\right]+\frac{\partial}{\partial z}\left[K(h) \frac{\partial h}{\partial z}+K(h)\right]-S(h)
$$

donde $\theta$ representa el contenido volumétrico de agua en el suelo $\left(\mathrm{cm}^{3} / \mathrm{cm}^{3}\right), t$ es el tiempo (día), x y z son las coordenadas espaciales horizontal y vertical (cm), respectivamente, $h$ el potencial de presión de agua en el suelo $(\mathrm{cm}), K(h)$ la función conductividad hidráulica insaturada (cm/día) y $S(h)$ el término sumidero de la ecuación $\left(\mathrm{cm}^{3} / \mathrm{cm}^{3} /\right.$ día), para considerar la extracción de agua por la planta.

Las funciones de retención de agua en el suelo y de conductividad hidráulica se determinaron según el modelo de van Genuchten-Mualem (van Genuchten, 1980). Los parámetros de las ecuaciones se indican en la Tabla 2 y fueron estimados con Rosetta (Schaap et al., 2001) a partir de los porcentajes de arena, limo y arcilla, densidad aparente y contenido de agua a -33 y $-1500 \mathrm{kPa}$ (Tabla 1).

Tabla 2. Parámetros de las ecuaciones de van Genuchten-Mualem utilizados en las simulaciones.

\begin{tabular}{|c|r|r|r|}
\hline Parámetro para la ecuación & $\begin{array}{r}\text { Franco- } \\
\text { arenosa }\end{array}$ & Franca & \multicolumn{1}{c|}{$\begin{array}{l}\text { Arcillo- } \\
\text { limosa }\end{array}$} \\
\hline Contenido residual, $\theta_{\mathrm{r}}\left(\mathrm{cm}^{3} / \mathrm{cm}^{3}\right)$ & 0,048 & 0,048 & 0,094 \\
\hline Contenido saturación, $\theta_{\mathrm{s}}\left(\mathrm{cm}^{3} / \mathrm{cm}^{3}\right)$ & 0,384 & 0,396 & 0,522 \\
\hline Alfa, $\alpha\left(\mathrm{cm}^{-1}\right)$ & 0,030 & 0,017 & 0,004 \\
\hline $\mathrm{n}(-)$ & 1,389 & 1,335 & 1,416 \\
\hline $\begin{array}{c}\text { Conductividad hidráulica a } \\
\text { saturación, } K_{s}(\mathrm{~cm} / \text { día })\end{array}$ & 32,32 & 20,44 & 15,66 \\
\hline
\end{tabular}

Se consideró un dominio de flujo de $100 \mathrm{~cm}$ de profundidad y $33 \mathrm{~cm}$ de anchura, desde la vertical donde se encontraba el lateral de riego hasta la posición intermedia entre dos laterales. La discretización espacial se realizó con elementos triangulares de dimensión cercana a $0,1 \mathrm{~cm}$ en la zona más próxima al emisor hasta aproximadamente $2 \mathrm{~cm}$ en la zona 
más alejada. En todas las simulaciones la condición inicial en todo el dominio fue de potencial matricial de $-33 \mathrm{kPa}$, correspondiente a las condiciones en el ensayo de campo en el suelo de textura franco-arenosa. Para poder comparar los resultados en los otros escenarios simulados se tomaron las mismas condiciones iniciales. Las condiciones de contorno fueron de ausencia de flujo en los dos laterales del dominio, gradiente unitario de potencial en el fondo, ya que de acuerdo a las lecturas de los piezómetros la profundidad del acuífero fue siempre superior a $2 \mathrm{~m}$, y una condición de contorno atmosférica en la superficie. En los elementos que representan el emisor se consideró una condición de flujo variable en el tiempo, en la que el caudal del emisor durante periodos de riego se obtuvo según:

$$
F=\frac{q}{d 2 \cdot \pi \cdot r}=\frac{24000 \frac{\mathrm{cm}^{3}}{\mathrm{dia}}}{30 \cdot 2 \cdot \mathrm{cm} \pi \cdot 0,85 \mathrm{~cm}}=149,8 \frac{\mathrm{cm}}{\text { día }}
$$

siendo $F$ el flujo aplicado en la zona del emisor ( $\mathrm{cm} / \mathrm{día}) ; q$ el caudal del emisor ( $\left.\mathrm{cm}^{3} / \mathrm{día}\right) ; d$ la separación entre emisores $(\mathrm{cm})$ y $r$ el radio exterior de los laterales de riego $(\mathrm{cm})$.

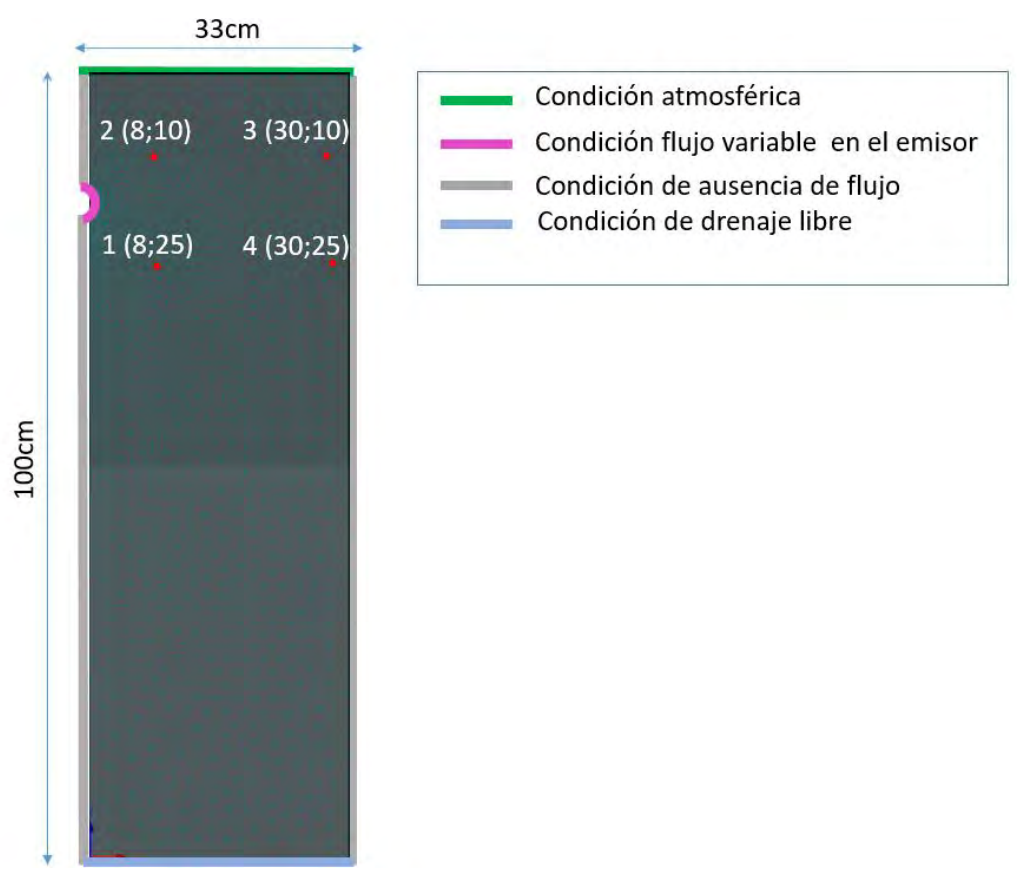

Figura 1. Dominio de flujo, posiciones de los sensores de contenido de agua $(1,2,3$ y 4) y condiciones de contorno. Los valores entre paréntesis indican la distancia horizontal respecto al lateral y la profundidad de los puntos, donde se monitorizó el contendido de agua.

Además de simular el ensayo de campo, se contemplaron los siguientes escenarios, que quedan recogidos en los casos A-V de la Tabla 2: 2 profundidades de los laterales de riego $(15$ y $25 \mathrm{~cm})$ y 3 texturas de suelo distintas (arcillo-limosa, franca y franco-arenosa), característica de tres suelos de la zona arrocera del Baix Ter. Cabe señalar que las profundidades consideradas para los laterales de riego son compatibles con las labores culturales previstas en el cultivo.

Las pautas de riego simuladas fueron: (1) pauta de riego practicado durante la campaña 2019 (casos A-C, Tabla 2); (2) riego basado estrictamente en la evapotranspiración del cultivo aplicando 3 frecuencias de riego distintas: 2 riegos al día, 1 riego diario y 1 riego cada 4 días (Casos D-V,Tabla 2).

Se consideraron los datos climáticos correspondientes a la temporada de riego 2019 desde la siembra (28/05) a la cosecha (18/10). La evapotranspiración del cultivo $\left(E T_{c}\right)$ se determinó según la ecuación: 


$$
E T_{c}=E T_{0} \cdot K_{c}
$$

siendo el $K_{c}$ el coeficiente de cultivo que se determinó para la zona de referencia a partir de los valores sugeridos por la FAO (Allen et al., 2006).

El código HYDRUS requiere introducir la evaporación y transpiración como dos términos independientes, ya que la evaporación se trata como una condición de contorno en superficie y la transpiración como el término sumidero en la ecuación de Richards. Para un cultivo que no cubra la totalidad de la superficie, lo que sucedió entre el momento de siembra y 70 días después, la evapotranspiración potencial se divide entre la evaporación potencial $\left(E_{p}\right)$ y la transpiración potencial $\left(T_{p}\right)$. La partición de estos dos términos se realizó mediante el índice de área foliar (LAl), siguiendo el procedimiento descrito en el modelo SWATRE y utilizando las ecuaciones (Belmans et al., 1983):

$$
\begin{aligned}
& E_{p}=E T_{c} \cdot e^{-K_{g r} \cdot L A I} \\
& T_{p}=E T_{c}-E_{p}
\end{aligned}
$$

El coeficiente para la radiación solar global en arroz $\left(K_{g r}\right)$ se tomó de 0,3 de acuerdo a los trabajos de Phogat et al. (2010) y Li et al. (2014). Los valores del LAl se determinaron en función del estadio fenológico a partir de los valores medidos en la misma parcela y con la misma variedad de arroz durante la campaña de riego del año 2018. En los periodos simulados en los que no hubo cultivo se consideró que la evaporación potencial era igual a la $E T_{0}$.

La extracción de agua por el cultivo se determinó de acuerdo a las ecuaciones propuestas por Feddes (1982) y con los parámetros de respuesta optimizados para el cultivo del arroz obtenidos por Singh et al. (2003), y aplicados entre otros por Li et al. (2014). Se consideró que la extracción de agua se localizó en la profundidad donde se desarrolla el sistema radicular del arroz, siendo más intensa en la zona más superficial y disminuyendo hasta alcanzar la profundidad máxima de las raíces, que fue de $25 \mathrm{~cm}$, de acuerdo con las observaciones realizadas en ensayos de campo a lo largo de la campaña de riego.

\section{3.- Validación del modelo de simulación}

El modelo HYDRUS 2D se validó previamente comparando los contenidos de agua en el suelo simulados con los contenidos de agua medidos en la parcela experimental en las posiciones descritas en el apartado 3.1. La validación corresponde al periodo de la campaña de riego y después de la cosecha, desde el día 01/07/2019, cuando se instalaron los sensores, hasta el día 22/01/2020. Para cuantificar la bondad de predicción del modelo se calcularon la raíz del error cuadrático medio (RMSE) y el coeficiente de determinación $\left(R^{2}\right)$.

\section{4.- Balance de agua, relación entre evapotranspiración y entradas de agua y percolación a $0,3 \mathrm{~m}$ de profundidad}

La simulación de la dinámica de agua en el suelo en la parcela experimental y en los escenarios considerados permitió realizar el balance de agua durante todo el periodo de cultivo (desde el día de siembra hasta la recolección, un total de 144 días), considerando las entradas de agua, lluvia y riego, así como las salidas, drenaje por debajo de $1 \mathrm{~m}$ y la evapotranspiración del cultivo. Además, también se calculó: la variación de la reserva de agua en todo el perfil, el error en el balance de masas del modelo y el agua que drenó por debajo de $0,3 \mathrm{~m}$ de profundidad, que se consideró no aprovechable por el cultivo, debido a la profundidad del sistema radicular. También se determinó la relación entre la evapotranspiración calculada por el modelo y las entradas de agua en el perfil del suelo. Esta relación indicaría el porcentaje de agua aprovechable por la planta en relación a las 
aportaciones de agua por riego y lluvia, siendo deseable maximizar su valor para aumentar la eficiencia del uso del agua por la planta.

\section{4- Resultados y discusión}

\section{1.- Campaña de riego en la parcela experimental}

Se analiza en este apartado la idoneidad de la pauta de riego seguida durante la campaña de cultivo. De acuerdo con la Figura 2, se observa que el agua aportada por riego durante las 3 primeras semanas fue superior a la demanda evapotranspirativa. El motivo se encuentra en la necesidad de favorecer la germinación de las semillas después de la siembra. Del día 15 hasta el 70 DDS (12/06/2019 al 06/08/2019), se regó con el objetivo de mantener el contenido de agua en el sensor 2 (Figura 1) a $0,27 \mathrm{~cm}^{3} / \mathrm{cm}^{3}$, correspondiente a un potencial matricial de $-10 \mathrm{kPa}$, resultando que el agua de riego aplicada fue inferior a la demanda del cultivo (ETC) (Figura 2). Para corregir este problema, se modificó la pauta de riego desde el día 70 al 123, periodo en que se aportó una dosis de riego ligeramente superior a las extracciones determinadas a partir de la ETC y, consecuentemente, a partir del día 84 el riego acumulado volvió a igualar la ETc acumulada (Figura 2). A partir del día 123 y hasta el día 140 se dejó de regar ya que el cultivo alcanzó la madurez fisiológica y lo que interesaba era reducir la humedad del grano para poder cosechar lo antes posible.

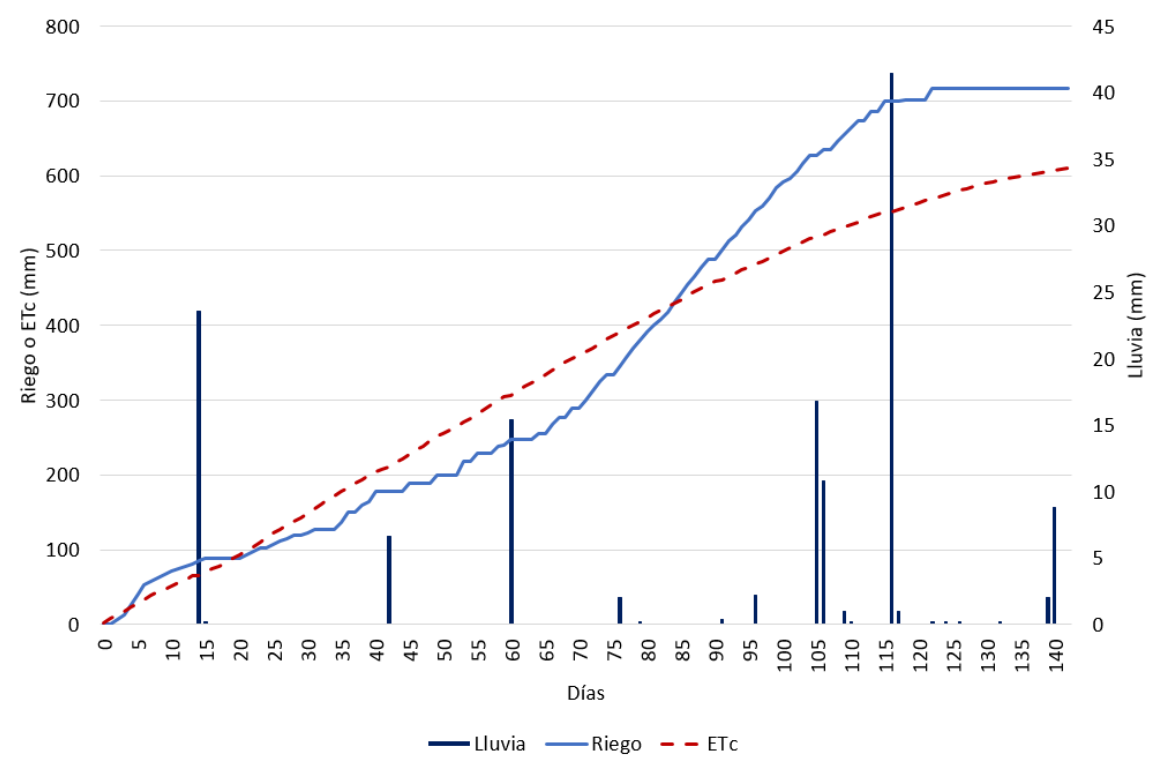

Figura 2. Riego y evapotranspiración del cultivo $\left(E T_{c}\right)$ acumulados a lo largo de la campaña de riego y eventos de precipitación.

Los contenidos de agua en el suelo observados en las distintas posiciones respecto al lateral de riego (Figura 1) se muestran en la Figura 3. El potencial matricial a $8 \mathrm{~cm}$ del lateral de riego y a $10 \mathrm{~cm}$ de profundidad se encontró por encima de $-10 \mathrm{kPa}$ (correspondiente a un contenido volumétrico de $0,27 \mathrm{~cm}^{3} / \mathrm{cm}^{3}$ ) durante todo el periodo de cultivo (de 34 a 140 días DDS), por lo que no debería suponer una reducción en la producción potencial del arroz (Bouman et al., 2005). A la misma distancia del lateral pero a $25 \mathrm{~cm}$ de profundidad, el potencial matricial estuvo por debajo de $-20 \mathrm{kPa}$ (correspondientes a $0,21 \mathrm{~cm}^{3} / \mathrm{cm}^{3}$ ) prácticamente durante todo el ciclo del cultivo. Con este valor son esperables reducciones notables de la producción (Cabangon et al., 2003).

En las posiciones más alejadas del lateral de riego (posiciones 3 y 4 ), el potencial matricial también estuvo por debajo de $-20 \mathrm{kPa}$ entre los días 34 y $70 \mathrm{DDS}$, coincidiendo con 
los días en que se regó con una dosis inferior a la $E T_{c}$, siendo esperable que el cultivo se viera gravemente afectado en la zona situada entre dos laterales de riego.

\section{2.- Validación del modelo}

La comparación de los contenidos de agua simulados y observados en la parcela experimental mostró una evolución similar, con valores de la raíz del error cuadrático medio (RMSE) de entre $0,028 \mathrm{~cm}^{3} / \mathrm{cm}^{3}$ y $0,064 \mathrm{~cm}^{3} / \mathrm{cm}^{3}$ y coeficientes de determinación $\left(R^{2}\right)$ entre 0,61 y 0,74 . Los valores de estos estadísticos son similares a los obtenidos en anteriores trabajos en los que se comparaba el contenido de agua observado y simulado, en suelo sin cultivo (Skaggs et al. 2004);en una plantación de manzanos (Arbat et al. 2008) y en experimentos a escala de laboratorio (Kandelous y Šimůnek 2010). En el presente estudio los RMSE más elevados se encuentran en la zona más alejada del lateral de riego, donde el contenido de agua simulado fue sistemáticamente superior al simulado con HYDRUS-2D. Estas diferencias podrían ser debidas a que el modelo no consideró la presencia de malas hierbas. En concreto, la población de Digitaria sp, que fue elevada durante y fuera del periodo de cultivo, y que, a diferencia del arroz, podrían continuar extrayendo agua a potenciales más bajos que los del arroz y dispone de un sistema radicular más profundo.
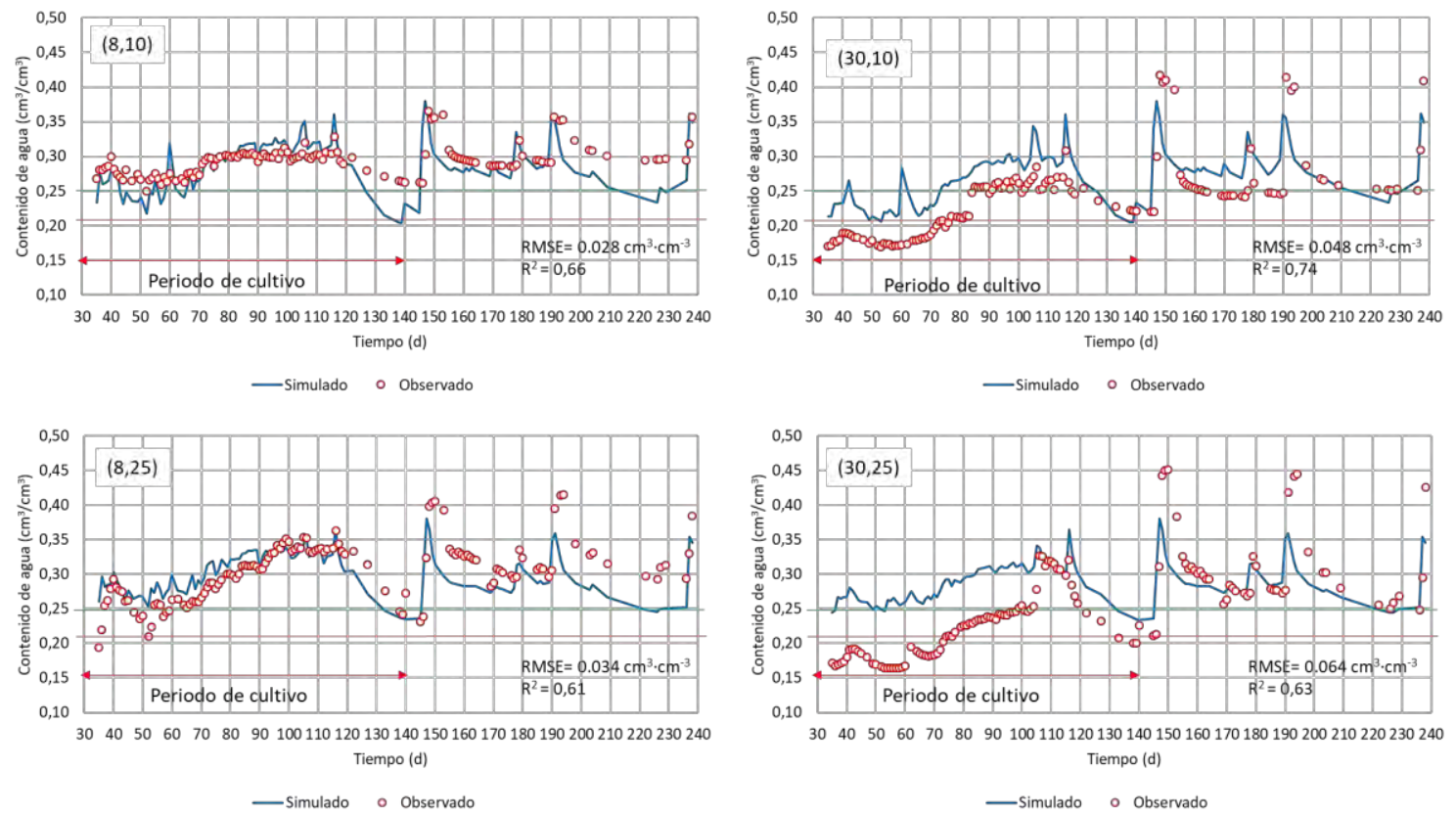

Figura 3. Comparación de los contenidos de agua simulados y observados en las 4 posiciones relativas al lateral de riego. En el eje horizontal la escala de tiempo indica los DDS, el 28/05/2019 corresponderia al día 0 . En el eje vertical se presenta el contenido volumétrico de agua correspondiente a: $-20 \mathrm{kPa}$ ; $\mathrm{y}-10 \mathrm{kPa}$

\section{3.- Componentes del balance de agua y percolación a $0,3 \mathrm{~m}$ de profundidad}

En la Tabla 1 se muestran las variables que determinan el balance de agua, la percolación por debajo de $0,3 \mathrm{~m}$, el error y algunas relaciones derivadas para cada uno de los casos analizados, tal como se describen en el apartado 3.4. 
Tabla 2. Componentes del balance de masas $(\mathrm{mm})$ simulados en $1 \mathrm{~m}$ de profundidad del perfil del suelo, error en el balance de masas $(\mathrm{mm})$, relación entre la $E T$ y las entradas de agua, y drenaje por debajo de la profundidad máxima de raíces $(0,3 \mathrm{~m})$.

\begin{tabular}{|c|c|c|c|c|c|c|c|c|c|c|c|c|}
\hline \multirow[b]{3}{*}{ O্ } & \multirow[b]{3}{*}{ 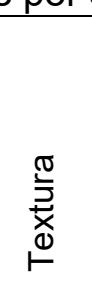 } & \multirow{3}{*}{ 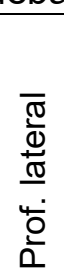 } & \multirow{3}{*}{\multicolumn{2}{|c|}{ 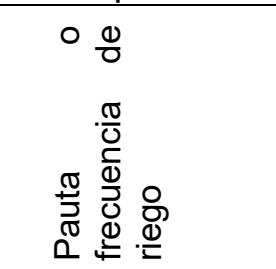 }} & & & & & & & & \\
\hline & & & & & \multicolumn{2}{|c|}{ Entradas } & \multicolumn{2}{|c|}{ Salidas } & \multirow[b]{2}{*}{ 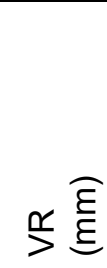 } & \multirow[b]{2}{*}{ 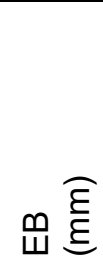 } & \multirow{2}{*}{ 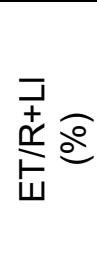 } & \multirow[b]{2}{*}{ 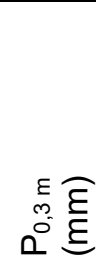 } \\
\hline & & & & & $\frac{\widehat{\xi}}{\stackrel{\widehat{\xi}}{\ltimes}}$ & $=\widehat{\widehat{\xi}}$ & 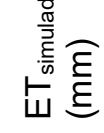 & 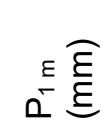 & & & & \\
\hline$A$ & 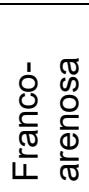 & 15 & \multirow{3}{*}{\multicolumn{2}{|c|}{ 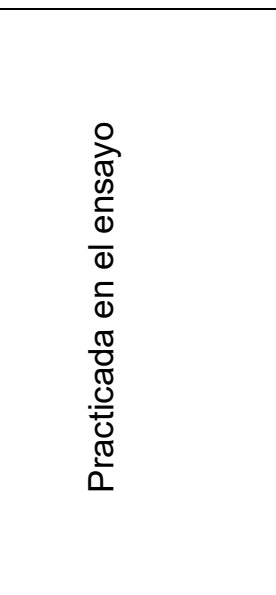 }} & 739 & 129 & -489 & -337 & -45 & -2 & 56 & 370 \\
\hline$B$ & $\begin{array}{l}\mathbb{\Xi} \\
\frac{0}{\pi} \\
\frac{\pi}{\leftarrow}\end{array}$ & 15 & & & 740 & 129 & -520 & -324 & -29 & -4 & 60 & 339 \\
\hline $\mathrm{C}$ & 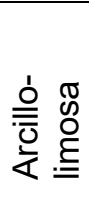 & 15 & & & 740 & 129 & -545 & -368 & 39 & -5 & 63 & 339 \\
\hline $\mathrm{D}$ & \multirow{6}{*}{ 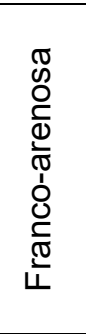 } & \multirow{3}{*}{15} & \multirow{18}{*}{$\frac{0}{\omega}$} & $\begin{array}{l}2 \text { veces al } \\
\text { día }\end{array}$ & 603 & 133 & -515 & -148 & -76 & -4 & 70 & 201 \\
\hline$E$ & & & & 1 vez por día & 603 & 132 & -511 & -148 & -77 & -2 & 70 & 201 \\
\hline $\mathrm{F}$ & & & & Cada 4 días & 604 & 126 & -491 & -162 & -77 & -1 & 67 & 215 \\
\hline$G$ & & 25 & & $\begin{array}{l}2 \text { veces al } \\
\text { día }\end{array}$ & 603 & 133 & -449 & -220 & -73 & -6 & 61 & 269 \\
\hline $\mathrm{H}$ & & $\angle 5$ & & 1 vez por día & 602 & 132 & -444 & -221 & -72 & -3 & 60 & 271 \\
\hline 1 & & & & Cada 4 días & 604 & 126 & -427 & -231 & -74 & -2 & 58 & 284 \\
\hline $\mathrm{J}$ & \multirow{6}{*}{ 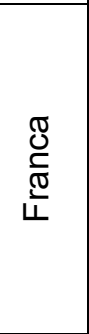 } & \multirow{3}{*}{15} & & $\begin{array}{l}2 \text { veces al } \\
\text { día }\end{array}$ & 603 & 133 & -553 & -129 & -59 & -5 & 75 & 170 \\
\hline $\mathrm{K}$ & & & & 1 vez por día & 606 & 132 & -549 & -128 & -60 & 0 & 74 & 168 \\
\hline $\mathrm{L}$ & & & & Cada 4 días & 604 & 126 & -533 & -137 & -60 & -1 & 73 & 178 \\
\hline$M$ & & & & $\begin{array}{l}2 \text { veces al } \\
\text { día }\end{array}$ & 603 & 133 & -511 & -176 & -57 & -8 & 70 & 213 \\
\hline $\mathrm{N}$ & & 25 & & 1 vez por día & 602 & 132 & -506 & -175 & -56 & -2 & 69 & 214 \\
\hline $\mathrm{O}$ & & & & Cada 4 días & 604 & 126 & -492 & -181 & -58 & -1 & 67 & 222 \\
\hline$P$ & \multirow{6}{*}{ 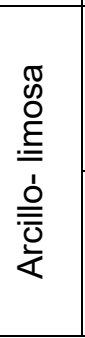 } & \multirow{3}{*}{15} & & $\begin{array}{l}2 \text { veces al } \\
\text { día }\end{array}$ & 603 & 133 & -544 & -221 & 17 & -12 & 74 & 209 \\
\hline Q & & & & 1 vez por día & 603 & 132 & -544 & -218 & 15 & -12 & 74 & 205 \\
\hline $\mathrm{R}$ & & & & Cada 4 días & 604 & 126 & -537 & -213 & 15 & -5 & 74 & 201 \\
\hline$S$ & & & & $\begin{array}{l}2 \text { veces al } \\
\text { día }\end{array}$ & 603 & 133 & -536 & -229 & 17 & -12 & 73 & 214 \\
\hline $\mathrm{T}$ & & 20 & & 1 vez por día & 602 & 132 & -535 & -229 & 19 & -11 & 73 & 214 \\
\hline $\mathrm{V}$ & & & & Cada 4 días & 604 & 126 & -531 & -219 & 16 & -4 & 73 & 209 \\
\hline
\end{tabular}

R: Riego, LI: Lluvia, ET: Evapotranspiración simulada, $\mathrm{P}_{1 \mathrm{~m}}$ : Percolación a $1 \mathrm{~m}$ de profundidad; VR: Variación de la reserva (negativa cuando aumenta el contenido), EB: Error en el balance de masas, ET/(R+LL): Porcentaje que representa la evapotranspiración del cultivo simulada respecto a las entradas de agua en el suelo $(R+L I)$, $\mathrm{P}_{0,3 \mathrm{~m}}$ : Percolación por debajo de 0,3 m.

El error en el balance de masas $(E B)$ calculado como: [Entradas + Salidas $+V R$ ] en las simulaciones realizadas con HYDRUS 2D fueron mínimos, con un valor de $12 \mathrm{~mm}$ en el caso en el que fue máximo, que corresponde a un error porcentual, definido como $\left[\frac{E B}{R+L l} \cdot 100\right]$, inferior al 1,7\%. Estos valores son ligeramente inferiores a los publicados por Li et al. (2014), donde se empleó el modelo HYDRUS-1D en arroz regado por inundación. En general, los 
errores en el balance de masas fueron superiores en las simulaciones con suelo de textura arcillo-limosa.

La ETC acumulada calculada según Penman-Monteith a lo largo de los 240 días del ciclo de cultivo fue de $642 \mathrm{~mm}$ (Figura 2), mientras el valor simulado con HYDRUS-2D estuvo entre un 66 y un $86 \%$ con respecto a este valor. La ET simulada fue mayor en las texturas arcillo-limosa y franca que en la franco-arenosa y, en general, disminuyó al reducir la frecuencia entre riegos y la profundidad del emisor, especialmente en las texturas de suelo más ligeras. La mayor $E T$ simulada en el suelo con textura franca que en el suelo con textura franco-arenosa (casos A y B - Tabla 1) podría explicar la mayor producción obtenida en la parte del campo en la que el suelo tenia textura franca, tal y como se muestra en el apartado 4.4. Por otro lado, el agua que drena por debajo de $0,3 \mathrm{~m}$ de profundidad fue superior en el suelo con textura franco-arenosa que en los suelos con textura franca y arcillo-limosa, lo cual explica que la evapotranspiración simulada y la relación $E T /(R+L L)$ fuesen superiores.

Cuando se regó restituyendo la evapotranspiración del cultivo (casos D-V) y la profundidad del lateral fue de $15 \mathrm{~cm}(\mathrm{D}, \mathrm{E}, \mathrm{F}, \mathrm{J}, \mathrm{K}, \mathrm{L}, \mathrm{P}, \mathrm{Q}$ y $\mathrm{R}$ ), se observó un incremento en la proporción entre agua evapotranspirada con respecto al agua aportada $E T /(R+L L)$ en relación con la pauta y frecuencia de riego practicada en el ensayo experimental $(A, B, y)$ fuera cual fuera la textura o frecuencia de riego seguida. Esto indicaría que regar siguiendo el criterio de restituir la evapotranspiración hubiera mejorado el aprovechamiento del agua.

En relación con la frecuencia de riego seguida, prácticamente no tuvo una influencia en la $E T$ simulada en el caso de textura arcillo-limosa (P-V). En cambio, se observó un ligero incremento de la relación $E T /(R+L L)$ al aumentar la frecuencia de riego en las texturas franco-arenosa y franca, del mismo modo que el incremento de la frecuencia redujo el drenaje por debajo de los $0,3 \mathrm{~m}$ de profundidad. La menor capacidad de retención de agua de los suelos con textura más ligera explicaría los resultados obtenidos.

Cuando se comparan las 2 profundidades de lateral de riego, en un suelo de textura franco-arenosa, para una misma frecuencia de riego, se observa que la profundidad de 15 $\mathrm{cm}$ redujo el drenaje por debajo de los $30 \mathrm{~cm}$ e incrementó la relación $E T /(R+L L)$ y consecuentemente el aprovechamiento del agua por la planta. Esto también sucedió en los suelos con textura franca y arcillo-limosa, aunque en menor medida.

\section{4.-Producción y productividad del agua}

La producción en el campo de ensayo mostró dos zonas bien diferenciadas, que podrían explicarse por las diferencias en la textura del suelo existente en la parcela. En la zona de textura franco-arenosa la producción fue significativamente menor $(p<0,05)$ que en la zona con una textura franca, $734 \mathrm{~kg} / \mathrm{ha}$ y $5363 \mathrm{~kg} / \mathrm{ha}$, respectivamente. Como se comentó en el apartado 4.1, los contenidos de agua que se midieron en la parte de textura franco-arenosa, correspondieron a valores de potencial matricial de $-20 \mathrm{kPa}$, e incluso inferiores en la zona más alejada del lateral de riego, durante el periodo de 34 a 70 DDS, correspondiente a los estadios de macollamiento e inicio de floración. Debido a que este periodo resulta especialmente sensible a la falta de agua, esto explicaría la baja producción obtenida en las partes de la parcela con textura franco-arenosa. Además, la poca disponibilidad de agua en la zona situada entre laterales en el suelo franco-arenoso (posición 3 en la Figura 1), pudo dar una ventaja competitiva a las malas hierbas que tienen la capacidad de desarrollarse a pesar de disponer de poca agua en el suelo. Sin embargo, la producción de $5363 \mathrm{~kg} / \mathrm{ha}$ y la productividad del agua $\left(W P_{R+L I}\right)$ de $0,62 \mathrm{~kg} / \mathrm{m}^{3}$ en la zona de textura franca, es similar a la que se obtuvo en ensayos de campañas anteriores utilizando el riego por goteo superficial en un suelo con textura arcillo-limosa y un acuífero muy somero (Tabla 3 ), en la que se obtuvieron $5565 \mathrm{~kg} / \mathrm{ha}$ y una $W P_{R+L /}$ de $0,60 \mathrm{~kg} / \mathrm{m}^{3}$ (Arbat et al., 2018).

En la Tabla 3 se muestran las productividades del agua de riego en el ensayo actual en RGS y los resultados obtenidos en riego por goteo superficial y enterrado durante la campaña 2017 en la misma zona (Arbat et al., 2018). 
Tabla 3. Producción, productividad del agua de riego $\left(W P_{R}\right)$ y de lluvia y riego $\left(W P_{R+L I}\right)$ en riego RGS, riego por goteo superficial y riego por inundación

\begin{tabular}{|l|c|c|c|}
\hline & Producción (kg/ha) & $W P_{R}$ & $W P_{R+L I}$ \\
\hline $\begin{array}{l}\text { RGS-2019 (Ensayo } \\
\text { actual) }\end{array}$ & 5363 & 0,73 & 0,62 \\
\hline $\begin{array}{l}\text { Riego por goteo-2017 } \\
\text { (Arbat et al., 2018) }\end{array}$ & 5565 & 0,72 & 0,60 \\
\hline $\begin{array}{l}\text { Riego por inundación } \\
2017 \text { (Arbat et al., 2018) }\end{array}$ & 6486 & 0,46 & 0,42 \\
\hline
\end{tabular}

En riego por inundación continua y siembra en seco los mismos autores reportaron una producción de $6486 \mathrm{~kg} / \mathrm{ha}$ y una $W P_{R+L /}$ de $0,42 \mathrm{~kg} / \mathrm{m}^{3}$. Aunque en riego por inundación la producción fue un $16 \%$ superior al RGS, la WP fue similar a la del riego por goteo superficial y representó un incremento de más del $40 \%$ en la $W P_{R+L}$ respecto al riego por inundación, por lo que el sistema de riego por goteo enterrado podría tener interés en situaciones donde el agua es un factor limitante y los suelos no son salinos.

Entre los pocos trabajos publicados de arroz regado por RGS, Rajwade et al., (2018) en un suelo de textura franco-arenosa obtuvieron producciones de entre 1881 y $5074 \mathrm{~kg} / \mathrm{ha}$ y $W P_{R+L /}$ entre 0,23 y $0,61 \mathrm{~kg} / \mathrm{m}^{3}$. Parthasarathi et al. (2018) en un suelo de textura arcillo-limosa obtuvo producciones de $5600 \mathrm{~kg} / \mathrm{ha}$ con $W P_{R+L I}$ de $0,86 \mathrm{~kg} / \mathrm{m}^{3}$. Si bien las producciones máximas reportadas por estos autores fueron muy similares entre sí y con la obtenida en el presente trabajo, existe una gran diferencia entre los valores de WP, que podrían ser debidas a la textura del suelo, ya que en general los suelos con mayor contenido en arena requieren dotaciones de riego superiores para que el agua del procedente del emisor pueda llegar al sistema radicular.

\section{5- Conclusiones y Recomendaciones}

Para aprovechar al máximo el potencial del riego por goteo subterráneo es necesario optimizar el diseño y los parámetros de manejo, destacando entre estos la profundidad de los emisores y la frecuencia de riego. Los valores del RMSE y $\mathrm{R}^{2}$ obtenidos comparando los contenidos de agua medidos en campo y simulados con HYDRUS-2D mostraron una predicción razonablemente buena, indicando que las simulaciones numéricas pueden ser:

(1) Una herramienta útil para optimizar el diseño y el manejo en RGS teniendo en cuenta las propiedades hidráulicas de los suelos, así como, (2) Predecir la percolación de agua. Los resultados de las simulaciones aplicadas al cultivo del arroz en la zona del Baix Ter en todas las texturas de suelo consideradas permiten establecer que la profundidad más adecuada para minimizar las pérdidas de agua por percolación y maximizar la extracción de agua por el cultivo es la de $15 \mathrm{~cm}$ con una frecuencia de riego elevada, de uno o dos riegos por día.

Por otro lado el criterio de riego basado en un umbral de potencial matricial presenta un punto crítico en la situación del punto de medida. Efectivamente, la gestión del riego basada en mantener un determinado potencial/contenido de agua en una posición demasiado cercana al lateral portaemisores condujo a un riego insuficiente. En este sentido las simulaciones con HYDRUS-2D podrían resultar muy útiles para determinar la localización más apropiada de los sensores para poder gestionar de forma eficiente el RGS en arroz.

\section{6.- Agradecimientos}

Los autores quieren agradecer al programa Europeo para la Investigación e Innovación Horizonte 2020- PRIMA, la financiación recibida en el marco del proyecto titulado "Hacia un uso sostenible del agua en los agro-ecosistemas Mediterráneos basados en el arroz (MEDWATERICE)", con referencia: PCl2019-103738. Asimismo, quieren agradecer a la empresa NETAFIM el apoyo técnico recibido en la realización del presente ensayo. 


\section{7- Bibliografía}

Allen, R. G., Pereira, L. S., Raes, D., \& Smith, M. (2006). Evapotranspiración del cultivo Guías para la determinación de las necesidades de agua de los cultivos. Estudio FAO Riego y Drenaje 56. FAO, Roma.

Arbat, G., Parals, S., Duran-Ros, M., Pujol, J., Puig-Bargués, J., \& Ramírez de Cartagena, F. (2018). Dinámica del agua en el suelo, productividad del agua y economía en riego por inundación y goteo en arroz. 19(1), 1-10. https://doi.org/10.25028/cnriegos.2018.a19

Arbat, G., Puig-Bargués, J., Barragán, J., Bonany, J., \& Ramírez de Cartagena, F. (2008). Monitoring soil water status for micro-irrigation management versus modelling approach. Biosystems Engineering, 100(2), 286-296. https://doi.org/10.1016/j.biosystemseng.2008.02.008

Belmans, C., Wesseling, J. G., \& Feddes, R. A. (1983). Simulation model of the water balance of a cropped soil: SWATRE. Journal of Hydrology, 63(3-4), 271-286. https://doi.org/10.1016/0022-1694(83)90045-8

Bouman, B. A. M., Peng, S., Castañeda, A. R., \& Visperas, R. M. (2005). Yield and water use of irrigated tropical aerobic rice systems. Agricultural Water Management, 74(2), 87105. https://doi.org/10.1016/j.agwat.2004.11.007

Cabangon, R., Lu, G., Tuong, T. P., Bouman, B. A. M., Feng, Y., \& Zhang, Z. C. (2003). Irrigation Management Effects on Yield and Water Productivity of Inbred and Aerobic Rice Varieties in Kaifeng. Proceedings of the 1st International Yellow River Forum on River Basin Management, Vol li, 65-75.

Kandelous, M. M., \& Šimůnek, J. (2010). Comparison of numerical, analytical, and empirical models to estimate wetting patterns for surface and subsurface drip irrigation. Irrigation Science, 1-10.

Li, Y., Šimůnek, J., Jing, L., Zhang, Z., \& Ni, L. (2014). Evaluation of water movement and water losses in a direct-seeded-rice field experiment using Hydrus-1D. Agricultural Water Management, 142, 38-46. https://doi.org/10.1016/j.agwat.2014.04.021

MAPA. (2020). Encuesta sobre superfícies y rendimientos de cultivos 2019. Ministerio de Agricultura, Pesca y Alimentación, Madrid.

Phogat, V., Yadav, A. K., Malik, R. S., Kumar, S., \& Cox, J. (2010). Simulation of salt and water movement and estimation of water productivity of rice crop irrigated with saline water. Paddy and Water Environment, 8(4), 333-346. https://doi.org/10.1007/s10333010-0213-7

Reyes-Esteves, R. G., \& Slack, D. C. (2019). Modeling Approaches for Determining Appropriate Depth of Subsurface Drip Irrigation Tubing in Alfalfa. Journal of Irrigation and Drainage Engineering, 145(10), 1-7. https://doi.org/10.1061/(ASCE)IR.19434774.0001409

Schaap, M. G., Leij, F. J., \& van Genuchten, M. T. (2001). Rosetta: a computer program for estimating soil hydraulic parameters with hierarchical pedotransfer functions. Journal of Hydrology, 251(3-4), 163-176. https://doi.org/10.1016/S0022-1694(01)00466-8

Šimůnek, J., van Genuchten, M. T., \& Šejna, M. (2008). Development and Applications of the HYDRUS and STANMOD Software Packages and Related Codes. Vadose Zone Journal, 7(2), 587. https://doi.org/10.2136/vzj2007.0077

Skaggs, T. H., Trout, T. J., Simunek, J., \& Shouse, P. J. (2004). Comparison of HYDRUS-2D Simulations of Drip Irrigation with Experimental Observations. En Journal of Irrigation and Drainage Engineering (Vol. 130, Número 4, pp. 304-310).

van Genuchten, M. T. (1980). A closed-form equation for predicting the hydraulic conductivity of unsaturated soils. En Soil Science Society of America Journal (Vol. 44, Número 5, pp. 892-898).

Zheng, J., Wang, W., Ding, Y., Liu, G., Xing, W., Cao, X., \& Chen, D. (2020). Assessment of climate change impact on the water footprint in rice production: Historical simulation and future projections at two representative rice cropping sites of China. Science of the Total Environment, 709, 136190. https://doi.org/10.1016/j.scitotenv.2019.136190 